\title{
A rare case of penile cancer in situ metastasizing to lymph nodes
}

\author{
Brian Kim; ${ }^{*}$ Francisco Garcia; ${ }^{\dagger}$ Naji Touma, MD; ${ }^{\neq \xi}$ Madeleine Moussa, MD, FRCPC; $;$ \\ Jonathan I. Izawa, MD, FRCSC ${ }^{\dagger \xi^{* *}}$
}

\begin{abstract}
Penile carcinoma in situ, or Queyrat's erythroplasia, is a rare condition of the glans penis. This lesion has been associated with invasive squamous cell carcinoma; however, metastasis without an invasive component is extremely rare. There have only been 2 documented cases with metachronous metastases. We report a third case in which metastases were diagnosed at presentation. The patient was a 51-year-old man who presented with a glans penile lesion and bilateral inguinal masses later determined to be carcinoma in situ with metastases to the inguinal and pelvic lymph nodes. He subsequently underwent a partial penectomy and lymphadenectomy followed by adjuvant chemotherapy and radiation. This case is discussed, along with a brief review of the literature.
\end{abstract}

CUAJ 2007;1(4):404-7

\section{Case Report}

A 51-year-old man of European descent presented with bilateral inguinal masses and a lesion at the meatus on the glans penis of several years duration for which he had not previously sought medical attention. The inguinal masses were noted during a cardiac catheterization procedure after the patient suffered a myocardial infarction. Ultrasound confirmed this to be lymphadenopathy. The patient underwent a right-sided excisional lymph node biopsy, which revealed moderately to poorly differentiated non-small cell carcinoma with squamous differentiation. CT scan of the abdomen and pelvis later showed bilateral inguinal lymphadenopathy; the largest node was on the left side and measured $5.7 \mathrm{~cm}$ in maximal diameter. There was no evidence of metastasis on chest radiography or bone scan. The patient did not have any constitutional symptoms. He had a medical history of ischemic heart disease, hypertension, chronic smoking and depression. He was then referred to our centre.

On physical exam, the patient was moderately overweight. His abdomen had a scar and there were fibrotic changes in the right inguinal region over the biopsy site. There was a large mass in the left inguinal area. On genitourinary exam, the patient's penis was uncircumcised and there was a $1.2 \times 1.5-\mathrm{cm}$ sessile, solid erythematous lesion around the urethral meatus (Fig. 1). The remainder of the exam was unremarkable.

The patient underwent a partial penectomy and bilateral superficial inguinal, deep inguinal and pelvic lymph node dissections. There was significant lymphadenopathy in the left inguinal region involving skin, which required a complete resection of the overlying skin. An abdominal fasciocutaneous flap was used for the resulting left inguinal defect.

Pathological review demonstrated carcinoma in situ of the glans penis (Fig. 2 and Fig. 3). There was no evidence of an invasive component and resection margins were negative. Five lymph nodes were positive for metastatic squamous cell carcinoma: a left and a right superficial inguinal node, a left deep inguinal node, and a left and a right pelvic node (Fig. 4). The patient was diagnosed with T3 N3 penile carcinoma in situ.

Two months after surgery, the patient underwent a 6-week course of concurrent cisplatin chemotherapy and external beam radiation to the pelvis. His post-treatment period has been complicated by significant bilateral scrotal edema. Follow-up has consisted of 3-month CT scans and the patient has been clinically disease-free 6 months after completing therapy.

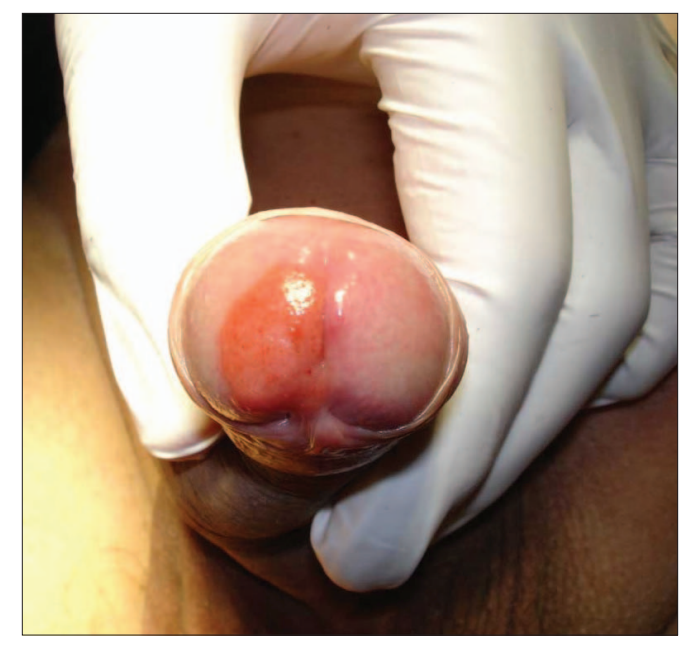

Fig. 1. This photograph shows carcinoma in situ of the glans penis (Queyrat's erythroplasia). 


\section{Discussion}

Penile carcinoma is an uncommon condition, accounting for less than $1 \%$ of all male cancers. ${ }^{1}$ Possible etiologies include human papilloma virus infection, phimosis, poor hygiene and cigarette smoking. ${ }^{2-5}$ Several precancerous penile lesions have been associated with invasive squamous cell carcinoma, including Queyrat's erythroplasia. This is a carcinoma in situ of the glans penis, first described by Queyrat in 1911 as a red, velvety, slightly-raised lesion that is sometimes ulcerated and papillary. ${ }^{6}$

Metastasis from penile carcinoma in situ is extremely rare, as there have only been 2 other reported cases., ${ }^{7.8}$ Avrach and Christensen described an aggressive case of penile carcinoma in situ. ${ }^{7}$ The patient underwent circumcision and inguinal lymph node dissections that later revealed cancerous tissue. ${ }^{7}$ Metastasis to lumbar paravertebral lymph nodes, liver and lumbar vertebrae were later discovered post mortem. ${ }^{7}$ Eng and colleagues reported another case of a similar lesion with metastasis to the inguinal and para-aortic lymph nodes. ${ }^{8}$ The patient was initially treated with circumcision and topical 5-fluorouracil until metastatic disease was discovered several years later, for which he underwent systemic chemotherapy and an inguinal lymph node dissection. ${ }^{8}$ The detection of metastatic disease from carcinoma in situ is therefore often incidental or delayed.

Several other factors are responsible for treatment delays and diagnostic challenges observed in penile cancer. Penile lesions are often poorly characterized, morphologically, making it difficult to differentiate between benign and atypical, and low- and high-grade lesions. ${ }^{2,9}$ Further, the natural progression of carcinoma in situ to invasive squamous cell carcinoma remains controversial. ${ }^{2}$ Graham and Helwig reported the potential for malignant invasion of Queyrat's erythroplasia as $10 \%$ based on 100 cases, with only 2 progressing to malignant disease. ${ }^{10}$ Patient factors are also largely responsible for postponed treatment. Compared with patients with other cancer types, penile cancer patients have been shown to defer medical care, possibly owing to embarrassment, fear or personal neglect. ${ }^{11}$ In the presenting case, the penile lesion and inguinal masses developed over several years before the patient sought medical attention.
There have been few reports of other noninvasive carcinomas with metastasis, and there have been few theories to explain this unusual occurrence. Rosen reported 8 cases of breast ductal and (or) lobular carcinoma in situ with axillary lymph node involvement. ${ }^{12}$ He postulated that obscure foci of invasion may have been missed through random tissue sampling despite vigorous histological analysis. Further, Ozzello and Sampitak demonstrated invasion by electron

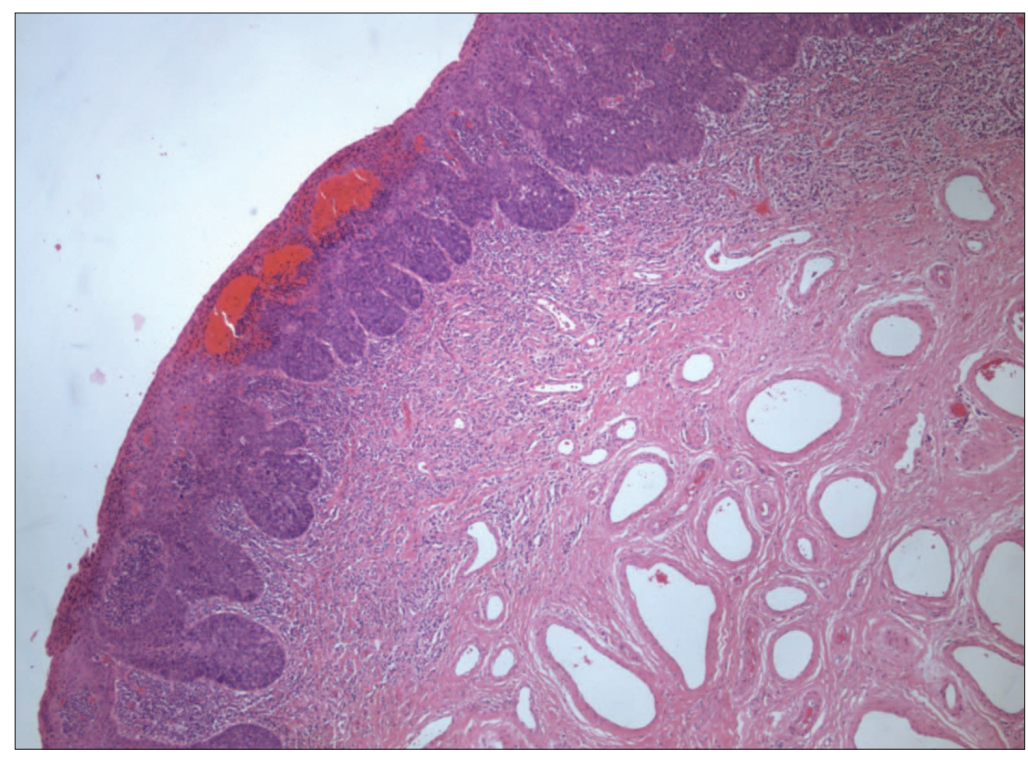

Fig. 2. Histopathology of the primary penile lesion showing carcinoma in situ of the glans penis (hematoxylin-eosin staining, original magnification $20 \times$ ).

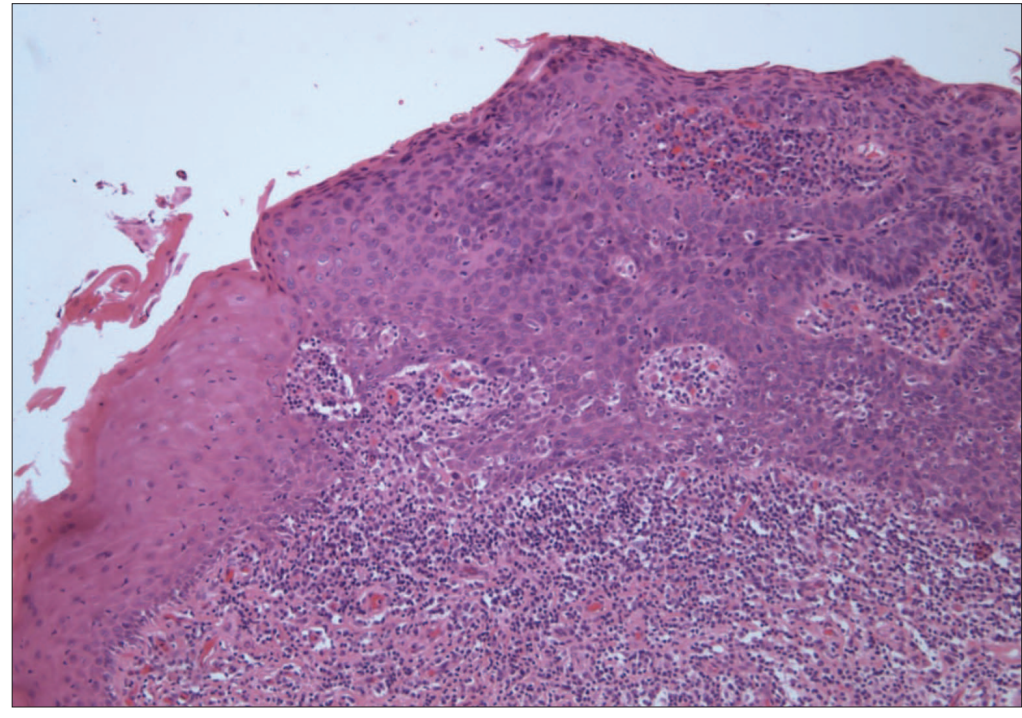

Fig. 3. Histopathology of the primary penile lesion showing carcinoma in situ of the glans penis (hematoxylin-eosin staining, original magnification $40 \times$ ). 


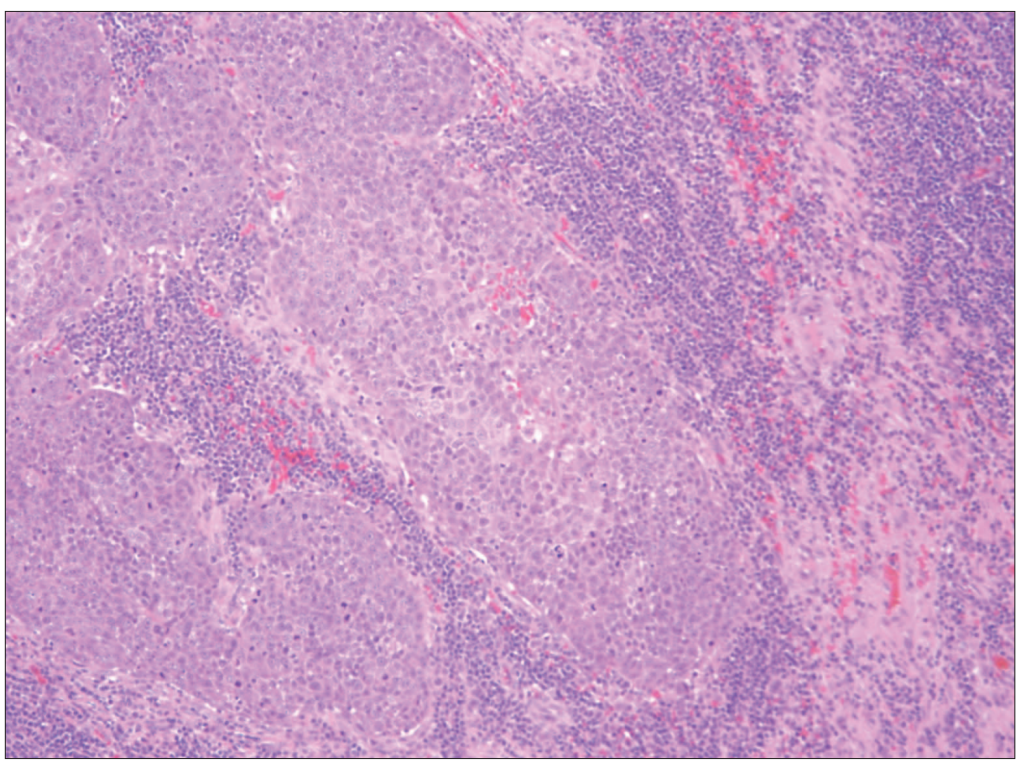

Fig. 4. Histopathology of the metastatic squamous cell carcinoma of an inguinal lymph node (hematoxylin-eosin staining, original magnification $40 \times$ ).

microscopy of ultrathin tissue sections that appeared only as carcinoma in situ on light microscopy. ${ }^{13}$ They concluded that light optic analysis alone may be limited in detecting early invasive disease. Our patient's cancer, however, was longstanding so that we would expect to identify invasion on standard histological exam. Sampling error was also negligible since the lesion was small and had negative resection margins. Therefore, there are likely other unidentified possibilities to account for metastasis without evidence of invasion.

There has been significant research into the "metastatic cascade," or the progression of a primary tumour to distant organs. ${ }^{14}$ Fidler $^{14}$ modernized Paget's $\mathrm{s}^{15}$ historic "seed and soil" hypothesis into 3 principles:

1. Neoplasms are composed of a biologically heterogeneous population, both genotypically and phenotypically.

2. Metastasis is a selective process for tumour cells that can successfully migrate to distant sites and proliferate.

3. Metastasis occurs preferentially in homeostatic environments that support the multiple interactions between the "seed" (tumour cell) and "soil" (organ).

Fidler's final principle may best explain the phenomenon observed in our patient. One hypothesis is that at a point in time of the primary tumour's natural history, tumour cells may have invaded the dermis, migrated and successfully proliferated in the lymph nodes with preferential host factors. The metastatic deposits then progressed and lead to further metastases rather than there being any further persistent findings of local progression and metastases from the primary tumour. The elucidation of such interactions, or "cross talk," is ongoing and has the potential to revolutionize future cancer therapy.${ }^{14}$ Our case highlights the possible tumour-host interactions necessary in the tumour and host microenvironment for tumour metastases without signs of persistent local tumour progression.

\section{Conclusion}

The unfortunate nature inherent to penile carcino$\mathrm{ma}$ is that patients may have the disease for a long duration before it is diagnosed and treated. This is partly attributed to a lack of available information in preventative medicine, diagnostic challenges of distinguishing carcinoma in situ from other similar lesions or conditions, and individual patient factors, such as embarrassment. However, as with most cancers, the early detection of disease is essential since morbidity and mortality are significantly worse for patients with locally advanced or metastatic disease. Future research into the "metastatic cascade" may reveal other new mechanisms, particularly for distant tumour growth without the histologic findings of local invasion of the primary tumour. In the meantime, the identification of any suspicious penile lesion should be investigated and treated promptly, with further caution to the metastatic potential of in situ lesions.

From the *Department of Medical Education, Faculty of Medicine, University of Toronto, Toronto, Ont., the $\$$ Schulich School of Medicine and Dentistry, Faculty of Medicine, the †Department of Surgery, the §Division of Urology, the qDepartments of Pathology and ${ }^{* *}$ Oncology, University of Western Ontario, London Health Sciences Centre and London Regional Cancer Program, London, Ont.

This article has been peer reviewed.

Competing interests: None declared.

\section{References}

1. Persky L. Epidemiology of cancer of the penis. Recent Results Cancer Res 1977;60:97109. 
2. Gerber G. Carcinoma in situ of the penis. J Urol 1994;151:829-33.

3. Wiener IS, Walther PJ. The association of oncogenic human papillomaviruses with urologic malignancy. Surg Oncol Clin N Am 1995;4:257-76.

4. Pratt-Thomas HR, Heins HC, Latham E, et al. The carcinogenic effect of human smegma: an experimental study. Cancer 1956;9:671-80.

5. Harish K, Ravi R. The role of tobacco in penile carcinoma. Br J Urol 1995;75:375-7.

6. Queyrat L. Erythroplasie du gland. Bull Soc Franc Derm Syph 1911;22:378-81.

7. Avrach WW, Christensen HE. Metastasizing erythroplasia Queyrat. Report of a case. Acta Derm Venereol 1976;56:409-12.

8. Eng TY, Petersen JP, Stack RS, et al. Lymph node metastasis from carcinoma in situ of the penis: a case report. J Urol 1995;153:432-4.

9. Cubilla AL, Velasquez EF, Young RH. Epithelial lesions associated with invasive penile squamous cell carcinoma: a pathologic study of 288 cases. Int I Surg Pathol 2004:12:351-64.

10. Graham JH, Helwig EB. Erythroplasia of Queyrat: a clinicopathologic and histochemical study. Cancer 1973;32:1396-414.
11. Lynch HT, Krush AJ. Delay factors in detection of cancer of the penis. Nebr State Med J 1969;54:360-7.

12. Rosen PP. Axillary lymph node metastases in patients with occult noninvasive breast carcinoma. Cancer 1980;46:1298-306.

13. Ozzello L, Sampitak P. The epithelial-stromal junction of intraductal carcinoma of the breast. Cancer 1970;26:1186-98.

14. Fidler II. The pathogenesis of cancer metastasis: the 'seed and soil' hypothesis revisited. Nat Rev Cancer 2003;3:453-8.

15. Paget $\mathrm{S}$. The distribution of secondary growths in cancer of the breast. Lancet 1889;1:571-3.

Correspondence: Dr. Jonathan I. Izawa, Departments of Surgery and Oncology, Division of Urology, London Health Sciences Centre - Victoria Campus, 800 Commissioners Rd. E., Suite C3-120, London ON N6A 4G5; jonathan.izawa@hsc.on.ca
We accept events for the Calendar section. You may send them to us in the following ways:

\section{Email \\ journal@cua.org}

Fax

514 875-0205

Mail
CUÄIJ
1155 University Ave.,
Suite 1014
Montréal, QC H3B 3A7

